\section{Gender bias: track revisions and appeals}

I suggest that journals should collect data at each stage of the peer-review process to help identify the barriers to publication that women face (see also J. Lerback and B. Hanson Nature 541, 455-457; 2017). Author gender needs to be incorporated into data on the numbers of manuscripts sent out for review, resubmitted after revision, and appealed against, successfully or unsuccessfully, by rejected authors.

I conducted a literature survey of my field (HIV) and found that, in 2015-16, less than 10\% of papers in Nature $(4 / 17 ; 24 \%)$ and Science $(0 / 24 ; 0 \%)$ together had a woman as the senior corresponding author. Although this sample is small and taken over a short period, the result is surprising, given the large number of women who served as organizer or chair at every major meeting in this field during that time and who represented roughly half of all US National Institutes of Health HIV studysection chairs. There is evidently a significant pool of strong women scientists in the field.

Comparison of key-stage evaluation data for male and female lead authors on accepted and rejected papers could shed light on gender bias in publication. For example, a reluctance to appeal may be more common among women. Understanding whether such factors contribute to gender-biased outcomes should help to counteract them. Julie Overbaugh Fred Hutchinson Cancer Research Center, Seattle, Washington, USA. joverbau@fredhutch.org

\section{Gender bias: strategy to balance reviewers}

I have discovered a useful strategy to help counter gender bias in peer review - an obstacle that is particularly relevant to younger scientists (see also J. Lerback and B. Hanson Nature
541, 455-457; 2017).

When prospective referees decline an invitation to review a manuscript, many journals ask them to propose alternative reviewers. If I am asked to suggest people, I consciously nominate female colleagues for the task especially early-career researchers or junior faculty members.

If the editor takes my advice and invites the nominated reviewer, colleagues (especially younger ones) are delighted and enthusiastic, knowing that undertaking peer review is an essential component of career building and being accepted into the scientific community. Enzo Palombo Swinburne University of Technology, Hawthorn, Victoria, Australia. epalombo@swin.edu.au

\section{Don't discount all curcumin trial data}

We argue that the clinical potential of the spice extract curcumin should not be dismissed simply on the grounds that it yields confusing results in molecular drug screens (Nature 541, 144-145; 2017; see also K. M. Nelson et al. J. Med. Chem. http://doi.org/bw46; 2017).

Nelson and colleagues claim a lack of evidence for curcumin's therapeutic benefits "despite thousands of research papers and more than 120 clinical trials" (www.clinicaltrials.gov). However, a PubMed search under curcumin double-blind placebocontrolled clinical trial' yields 49 entries, of which 17 recent trials show efficacy. In addition, there are 27 other clinical trials and at least 5 animal studies of curcumin that point to therapeutic benefits (see full reference list online).

The assumption that a drug candidate must have a single known target and compatibility with high-throughput screening to enter the clinic can preclude promising drug candidates (R. L. Elliott Am. Chem. Soc. Med. Chem. Lett. 3, 688-690; 2012). Current detection methods for target engagement cannot gauge the full pharmacological spectrum of an investigational drug, so should be used with other screening paradigms. Also, the binding behaviour of curcumin to multiple molecular targets is associated with modulation rather than outright inhibition. And high-throughput screening is prone to technical artefacts that can make it a deceptive arbiter for excluding potential drugs.

In light of these considerations, curcumin's molecular targets and their regulatory mechanisms warrant further investigation if we are to build on the promising results that are already to hand in humans and animals.

Michal Heger ${ }^{*}$ University of Amsterdam, the Netherlands. m.heger@amc.uva.nl

${ }^{*}$ On behalf of 13 correspondents (see go.nature.com/2mrrxfh for full list and references).

\section{Three-point plan for reproducibility}

The success of clinical studies depends on the reproducibility of preclinical research results (see J. Kimmelman and C. Federico Nature 542, 25-27; 2017). We propose a three-tier principle of transparency, replication and triangulation that should be achieved before publication, to ensure that the results warrant further study in preclinical and clinical trials.

Transparency focuses on the availability of complete and clear information about experimental methodology. This must be sufficient to allow the published study to be replicated under the same conditions by other investigators, with essentially the same primary outcomes.

Replicability should be tested by the original researchers and/or by others in the same laboratory, and confirmed using different samples or specimens. Ideally, an unrelated lab should perform independent replication based on the reported methods.

Triangulation confirms the study's central findings or models using different methodologies and experiments, ensuring that measurements converge from different experimental perspectives. For example, Robert Edwards and colleagues studied maturation of in vitro fertilized embryos in rats, mice and hamsters before predicting the technique's success in humans.

In our view, fulfilling all three tiers is manageable and essential. In the long term, the guaranteed quality of the results will more than justify the delay to publication. E. Andrew Balas Augusta University, Georgia, USA. Lee M. Ellis University of Texas M.D. Anderson Cancer Center, Houston, Texas, USA. andrew.balas@augusta.edu

\section{Reformatting wastes public funds}

Limited public funds for scientific research are being spent on reformatting manuscripts for different journals, without any apparent gain for science or society (see Q. Guo Nature 540, 525; 2016 and J. P. Moore Nature

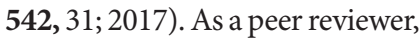
I am interested in a manuscript's content - not its format. The increasing popularity of preprint servers indicates that format does not bother readers either.

In 2013, for instance, Nature published less than $8 \%$ (856 of 10,952 ) of the research papers submitted (see go.nature. $\mathrm{com} / 2 \mathrm{~m} 102 \mathrm{lb})$. If it takes authors, say, an hour or more to reformat each rejected article for submission to another journal, this will amount to some 10,000 scientist-hours over just one year.

For many papers that are rejected without review, there will be no need to change the scientific content before resubmission, and so paid time spent on reformatting them is not even scientifically justified. Julian Budd Institute of Experimental Medicine, Budapest, Hungary. julian.budd@koki.mta.hu 